\title{
Quality of care measures for migraine: a comprehensive review
}

\author{
Joshua J. Gagne \\ Thomas Jefferson University \\ Brian Leas \\ Thomas Jefferson University \\ Jennifer H. Lofland \\ Thomas Jefferson University \\ Neil Goldfarb \\ Thomas Jefferson University \\ Frederick Freitag \\ Diamond Headache Clinic
}

Follow this and additional works at: https://jdc.jefferson.edu/healthpolicyfaculty

See next page for additional authors

Part of the Health Services Research Commons

Let us know how access to this document benefits you

\section{Recommended Citation}

Gagne, Joshua J.; Leas, Brian; Lofland, Jennifer H.; Goldfarb, Neil; Freitag, Frederick; and Silberstein, Stephen, "Quality of care measures for migraine: a comprehensive review" (2007). College of Population Health Faculty Papers. Paper 39.

https://jdc.jefferson.edu/healthpolicyfaculty/39

This Article is brought to you for free and open access by the Jefferson Digital Commons. The Jefferson Digital Commons is a service of Thomas Jefferson University's Center for Teaching and Learning (CTL). The Commons is a showcase for Jefferson books and journals, peer-reviewed scholarly publications, unique historical collections from the University archives, and teaching tools. The Jefferson Digital Commons allows researchers and interested readers anywhere in the world to learn about and keep up to date with Jefferson scholarship. This article has been accepted for inclusion in College of Population Health Faculty Papers by an authorized administrator of the Jefferson Digital Commons. For more information, please contact: JeffersonDigitalCommons@jefferson.edu. 
Authors

Joshua J. Gagne, Brian Leas, Jennifer H. Lofland, Neil Goldfarb, Frederick Freitag, and Stephen Silberstein 


\title{
Quality of Care Measures for Migraine: A Comprehensive Review
}

\author{
JOSHUA J. GAGNE, Pharm.D., ${ }^{1,2}$ BRIAN LEAS, M.A., B.A., ${ }^{1}$ \\ JENNIFER H. LOFLAND, Pharm.D., M.P.H., Ph.D., ${ }^{1}$ NEIL GOLDFARB, B.A., ${ }^{1}$ \\ FREDERICK FREITAG, D.O., ${ }^{3}$ and STEPHEN SILBERSTEIN, M.D. ${ }^{4}$
}

\begin{abstract}
Migraine headache is a highly prevalent, chronic, episodic disorder that is associated with high direct and indirect costs. Migraine headache impacts not only patients, but also their employers due to substantial decreases in workplace productivity. Despite the prevalence and clinical and economic burdens of migraine, no national efforts to develop and implement standardized measures of quality of care have been made. The objective of this study was to collect and report on existing quality of care measures for migraine that could be suitable for quality measurement at the health-plan level. Published literature, the Agency for Healthcare Research and Quality's National Quality Measure Clearinghouse, and resources available from quality organizations (eg, the National Committee for Quality Assurance) were examined to identify existing quality indicators that can be used to assess the quality of care delivered to migraine sufferers at the health-plan level. Among the results of the study were the following: Quality of care measures for migraine include patient-reported measures and non-patient reported, diagnosis-related, prevention-related, and treatment-related indicators. Most existing measures have been developed by the Institute for Clinical Systems Improvement or summarized and reported by the RAND Corporation. Few of these measures can be used to assess migraine quality of care at the health-plan level. In conclusion, many measures exist, but they are not intended for use at the health-plan level. Incorporation of valid and reliable quality of care measures may increase the ability of migraine disease management programs to conform to clinical care guidelines. Significant effort is needed to determine what and how to measure quality among health plans to improve the quality of care delivered to individuals with migraine. (Disease Management 2007;10:138-146)
\end{abstract}

\section{BACKGROUND}

T HOUGH EPISODIC IN NATURE, migraine is a highly prevalent ${ }^{1}$ and chronic disorder that is most prevalent among individuals in their fourth decade, ${ }^{2,3}$ a time when they are highly active in the professional arena. ${ }^{4}$ Direct and indirect costs associated with migraine are substantial. Estimates of direct health care costs range from $\$ \mathrm{US} 1.25$ billion $^{5}$ to $\$ 11.1$ billion. $^{6}$

\footnotetext{
${ }^{1}$ Department of Health Policy, Jefferson Medical College, Philadelphia, Pennsylvania.

${ }^{2}$ Ortho-McNeil Janssen Scientific Affairs, LLC, Titusville, New Jersey.

${ }^{3}$ Diamond Headache Clinic, Chicago, Illinois.

4Jefferson Headache Center, Thomas Jefferson University Hospital, Philadelphia, Pennsylvania.
} 
Migraine negatively impacts employers, with annual lost workplace productivity estimated to be in the range of $\$ 16.2$ billion ${ }^{5,7}$ to $\$ 28.7$ billion in 2003 dollars. ${ }^{5}$ The annual indirect burden of illness is estimated to be $\$ 2,834$ per patient. ${ }^{8}$ Estimates of workplace absenteeism due to migraine headache range from 1 to 7 days per year, ${ }^{9-12}$ and presenteeism, which is defined as being at work but less than $100 \%$ productive, constitutes $89 \%$ of the total cost burden among those with migraine. ${ }^{13}$

Despite the prevalence and substantial economic burden of migraine, no standardized measures of quality of care exist. The Health Plan Employer Data and Information Set, maintained by the National Committee for Quality Assurance (NCQA) and widely used to assess quality of care at the health-plan level, does not include any headache- or migraine-related measures. ${ }^{14}$ The National Quality Forum (NQF), which has recently merged with the National Committee for Quality Health Care, and the Ambulatory Care Quality Alliance (AQA) have not focused attention on quality of care for individuals with migraine headache. ${ }^{15,16} \mathrm{It}$ is not clear why these initiatives have not focused on migraine, but it may be due, in part, to a lack of understanding of migraine or its underdiagnosis. The American Migraine Prevalence and Prevention Study found that only $56.2 \%$ of those with migraine had ever received a medical diagnosis. ${ }^{2}$ Since many publications provide insights into the large clinical and economic burden of the disorder, ${ }^{1-13,17}$ patients, employers, disease management providers, and health plans alike will benefit from a standardized set of migraine quality measures. Indeed, employers have begun getting involved in the management of migraine in the workplace to help keep employees at work and to help them maintain effectiveness while working. These efforts are a means to reduce overall costs attributable to the disorder. ${ }^{18,19}$

Health plans and disease management providers also are getting involved in migraine care initiatives. Presently, 2 disease management organizations have received accreditation or certification by NCQA for migraine programs. ${ }^{14}$ Migraine disease management programs providing patient and physician educa- tion, migraine diaries, patient feedback reports, and access to telephonic disease management units have demonstrated effectiveness in improving migraine-specific humanistic outcomes, measured by the Migraine Therapy Assessment Questionnaire (MTQA) and the Migraine Disability Assessment (MIDAS) questionnaire. ${ }^{20,21}$ Integration of valid and reliable evidence- and consensus-based quality of care measures can assist health plans and disease management providers in better evaluating interventions and edging intervention and measurement activities closer to conformation with standard of care guidelines. The purpose of this review is to collect and report on existing quality of care measures for migraine that could be suitable for quality measurement at the health-plan level.

\section{TRANSLATION OF CLINICAL GUIDELINES INTO QUALITY INDICATORS}

A major milestone in migraine care was the establishment of the US Headache Consortium, which is composed of 7 organizations that have an interest in improving the quality of care for people with migraine disorders. ${ }^{22}$ Member organizations include the American Academy of Family Physicians, the American Academy of Neurology, the American Headache Society, the American College of Emergency Physicians, the American College of Physicians, the American Osteopathic Association, and the National Headache Foundation. The Consortium developed an evidence-based migraine practice parameter and, in doing so, identified acute and long-term treatment goals that included reducing attack frequency and severity, reducing disability, improving quality of life, preventing headache, avoiding headache medication escalation, and educating and enabling patients to manage their disease. ${ }^{22}$ To achieve these goals, the consortium developed the recommendations summarized in Table 1.

Translating the care delivered to individuals with migraine headache into measurable indicators of quality is challenging. The Institute of Medicine defines quality as, "The degree to which health services for individuals and pop- 
Table 1. US Headache Consortium Goals of Migraine Treatment and Prevention*

- Educate migraine sufferers about their condition and its treatment, and encourage them to participate in their own management.

- Use migraine-specific agents (ie, triptans, dihydroergotamine, ergotamine) for patients with more severe migraine and for those whose headaches are known to respond poorly to nonsteroidal anti-inflammatory drugs, or NSAID, or combinations such as aspirin plus acetaminophen plus caffeine.

- Select a non-oral route of administration for patients whose migraines present early with nausea or vomiting as a significant component of the symptom complex.

- Consider a self-administered rescue medication for patients with severe migraines that do not respond well to (or fail) other treatments.

- Guard against medication-overuse headache. ("Rebound headache" or "drug-induced headache" are sometimes used interchangeably with "medication over-use headache.")

- Preventive therapy in appropriately guided clinical presentations, including:

- Headache frequency more than 2 days per week (or $>8$ days/month).

- Use of acute medications, successfully or unsuccessfully, more than 2 days per week.

- Headache attacks that remain disabling despite aggressive acute intervention, as documented by lifestyle interference, ratings on disability scales, or use of rescue medications more than once a month.

- Presence of prolonged aura ( $>1$ hour), complex aura (basilar or hemiplegic), or migraine-induced stroke.

- Contraindications to, failure of, overuse of, or adverse events with acute therapies.

- Patient desire to reduce frequency of acute attacks.

*Adapted from the US Headache Consortium Guidelines ${ }^{22}$

ulations increase the likelihood of desired health outcomes and are consistent with current professional knowledge." ${ }^{23}$ Quality of care can be measured along any 1 of 3 domains. This "Quality Triad" includes structures (ie, the "bricks and mortar" of care delivery), processes (ie, the way care is delivered), and outcomes (ie, the results of care). ${ }^{24}$ An example of a process measure is using a beta-blocker after myocardial infarction in patients without contraindications to such therapy. Such an indicator of quality is supported by substantial clinical research and is now included in various quality measurement sets. More recently, attention has been given to the use of outcome measures as the basis for quality measurement and quality improvement. ${ }^{25}$ Outcome measures can be subdivided into clinical, humanistic, and economic measures. The measures selected should be professionally developed, with agreed-upon standards of care, and, most important, the measures must be interpretable and actionable.

\section{METHODS}

To develop a migraine quality measurement set that could be used at the health-plan level, we conducted a resource review including published peer-reviewed literature, the Agency for
Healthcare Research and Quality's National Quality Measure Clearinghouse, and resources available from quality organizations such as NCQA, NQF, and AQA. We grouped the identified measures as either (1) patient-reported or (2) non-patient-reported. Within the non-patientreported measures, there are (a) diagnosis-related, (b) treatment-related, and (c) preventionrelated measures. This scheme is a standard clinical approach and is similar to the structure of the US Headache Consortium guidelines. ${ }^{22}$

\section{RESULTS}

\section{Patient-reported measures}

Because of the subjective nature of migraine headaches, many measurement techniques exist that rely on patient-reported information. We briefly discuss each of these instruments below.

24-hour migraine quality of life questionnaire. The 24-Hour Migraine Quality of Life Questionnaire (24-Hr-MQOLQ) ${ }^{26,27}$ is designed to assess the health of specific populations at a specific point in time. It is a 15-item, self-administered questionnaire aimed at assessing migraine sufferers' quality of life within 24 hours of taking migraine medication and 
within the first 24 hours of a migraine attack. Items cover a number of domains including work, social, energy, vitality, feelings, concerns, and migraine symptoms.

Migraine-specific quality of life. The MigraineSpecific Quality of Life (MSQOL) ${ }^{28}$ is a 25 -item self-administered instrument developed to measure the long-term effects of migraine and migraine treatment on quality of life. Items are summed to provide an overall score and 3 subscores measuring physical, emotional, and social domains. A total MSQOL score also can be calculated.

Migraine-specific quality of life questionnaire, version 2.1. The Migraine-Specific Quality of Life Questionnaire, version 2.1 (MSQ v.2.1) 29,30 is a 14-item, self-administered instrument covering areas of specific concern to persons with migraine. Specifically, it addresses factors of role restrictive, role preventive, and emotional function. It was developed from the MSQ v.1.0 and subsequently shortened for easier administration; its content was improved by rewording certain items for greater clarification. The MSQ v.2.1 is intended to respond to a perceived need to develop a health status measure that would adequately focus on the physical and emotional limitations that are typically associated with migraine headaches.

Migraine disability assessment scale. Migraine Disability Assessment Scale (MIDAS) ${ }^{31,32}$ is a questionnaire that measures migraine-related disability based on the number of days of lost and limited activity due to migraine. Activities are classified into 3 areas, which include (1) paid work and education (school/college); (2) household work (unpaid work such as housework, shopping, and caring for children and others); and (3) family, social, and leisure activities. Patients with migraine count the number of days on which they missed out on activities because of migraine in the previous 3 months. They also count the number of days on which their productivity was, at most, half of normal in paid and household work. The overall MIDAS score is calculated by summing the answers to these 5 questions, and is scored in number of days.
Headache disability inventory. The Headache Disability Inventory (HDI) ${ }^{33,34}$ is a 25 -item instrument developed to quantify the impact of headache, though not specifically migraine, and its treatment on daily living. Items are dichotomized and answered with either "yes" (4 points), "sometimes" (2 points), or "no" (0 points). Based on a scale of $0-100$, the scores indicate patients' self-perceived headache disability. These measures assist the physician in determining the medical relevance of patients' headaches.

Headache impact questionnaire. The Headache Impact Questionnaire (HImQ) $)^{35,36}$ is a 16-item, self-administered questionnaire used to measure pain and activity limitations from headache, though not specifically migraine, over a 3 -month recall period. This instrument was developed to capture information about pain and headache-related disability to help doctors and patients evaluate the impact of headache on the individual's daily life. Headache-related disability is expressed as hours or days of lost productivity and functioning.

Headache impact test. The Headache Impact Test (HIT) ${ }^{37-39}$ is a tool that measures the impact headaches, though not specifically migraine, have on a person's ability to function on the job, at home, at school, and in social situations. The HIT builds on previous work by pooling items from 4 existing measurement instruments: MSQ, HDI, HIMQ, and MIDAS. Items cover a broader spectrum of health outcomes than any of the original scales, ranging from pain to emotional distress. Two versions exist, the computer adaptive testing (CAT)-HIT and the HIT-6. The CAT-HIT is administered over the Internet, whereas the HIT-6 is a static, short-form version of the HIT for paper-andpencil administration.

Migraine therapy assessment questionnaire. The Migraine Therapy Assessment Questionnaire $(\mathrm{MTAQ})^{40}$ is a 9-item, patient-completed questionnaire that was developed to identify individuals whose migraine management may be suboptimal. MTAQ was created in an effort to identify barriers to optimal migraine management and improve patient outcomes. 
Migraine assessment of current therapy. The Migraine Assessment of Current Therapy (Migraine-ACT $)^{41}$ is a 4 -item assessment tool intended to evaluate how well a recently prescribed acute medication is working, and to identify patients who require a change in acute treatment. The questions were formulated in 4 domains including headache impact, global assessment of relief, consistency of response, and emotional response.

In addition to patient self-report survey instruments, the Institute for Clinical Systems Improvement (ICSI) suggests some providerlevel humanistic indicators of patient functional status including percentage of days per month with migraine for migraine sufferers; hours (days) per month lost due to migraine for migraine sufferers; rate of emergency department usage by migraine sufferers for migraine; and percentage of migraine sufferers with a headache calendar or diary. ${ }^{42}$

ICSI is an independent, nonprofit organization that facilitates health care quality improvement initiatives among providers in Minnesota and surrounding areas. The organization develops diagnosis and treatment algorithms for various conditions, including headache, and establishes priority aims and suggested measures to improve the quality of care for these conditions. The seventh edition of the diagnosis and treatment of headache guidelines, released in January of 2006, includes indicators (with measurement specifications) of quality for headache at the provider level based on chart review. While these measures may be important to consider in developing a migraine-specific quality measure set, implementing such measures may be diffi- cult at the health-plan level because chart review may not be feasible for health plans. Furthermore, these types of measures may be suboptimal for disease management programs operating in collaboration with health plans because it would require program providers to cull additional data not available through the health plan, thereby increasing required resources and costs.

\section{NON-PATIENT-REPORTED MEASURES}

In 2003, McGlynn and colleagues at the RAND Cooperation published a landmark study which sought to quantify the extent to which quality health care was delivered in the United States. ${ }^{43}$ Among the 439 performance indicators included in the study, 21 were specific to headache (not specifically migraine). These indicators were derived from a literature review conducted by researchers at the RAND Corporation. ${ }^{44,45}$ The researchers conducted a MEDLINE search of English language articles between 1990 and 1997, using the keywords headache, diagnosis, and treatment. Of the 14 articles identified, 9 were review articles and 5 were observational studies. Each of the indicators that were collected was categorized as either a diagnosis indicator or a treatment indicator.

In the aforementioned article, McGlynn and colleagues classified all the measures in the comprehensive collection on a number of domains, including unit (ie, patient, dyad, episode); type (ie, preventive, acute, chronic); function (ie, screening, diagnosis, treatment, follow-up); modality (ie, history, physical ex-

Table 2. Diagnosis-Related Migraine Quality of Care Indicators*

- Patients with new onset headache should have an examination evaluating the cranial nerves.

- Patients with new onset headache should have an examination evaluating the fundi.

- Patients with new onset headache should have an examination evaluating deep tendon reflexes.

- Patients with new onset headache should have an examination evaluating their blood pressure.

- CT or MRI scanning is indicated in patients with new onset headache and an abnormal neurological examination.

- CT or MRI scanning is indicated in patients with new onset headache and a severe headache.

- Percentage of headache sufferers diagnosed with migraine.

- Percentage of headache sufferers diagnosed with tension-type headache.

- Percentage of headache sufferers diagnosed with cluster headaches.

- Percentage of headache sufferers diagnosed with sinus headaches.

CT, Computed tomography; MRI, Magnetic resonance imaging.

*Adapted from references $42,44,45$. 
amination, counseling/education, encounter or other intervention, medication, laboratory/ radiology, immunization); problem (ie, underuse, overuse); and level of evidence (ie, randomized trial, nonrandomized controlled study [eg, case control], observational study/ expert opinion). ${ }^{43}$ The rate of performance for each indicator was calculated to estimate the percentage of recommended care received by study participants. Mean scores for headache quality indicators ranged from $8.21 \%$ (patients with new-onset headache should be asked about family history of headache) to $100 \%$ (sumatriptan and ergotamine should not be given to patients with a history of ischemic heart disease or angina). In other words, on average, patients received recommended care between $8.21 \%$ to $100 \%$ of the time based on various indicators. Overall, recommended care for headache was received $45.2 \%$ of the time. ${ }^{43}$ Clearly, performance varied widely across different indicators of care. Of the 21 headache quality measures included in this study, the indicators that best lend themselves to measures rather than just guidelines are included in Tables 2 and 3. Because these indicators are for headache, they may not apply to migraine specifically.

All of the non-patient-reported measures are classified as either diagnosis-related $42,44,45$ (Table 2) or treatment- and preventionrelated $^{42,44-48}$ (Tables 3 and 4). The RAND authors assert that screening for headaches is not recommended because the problem is symptomatic. ${ }^{44,45}$ However, Lipton and colleagues developed the ID Migraine, a 3-item questionnaire used to screen for and diagnose migraine in the physician's office. ${ }^{50}$ Cady and others have developed similar questionnaires. ${ }^{51}$ The ICSI guidelines suggest that several measures could be used to increase the accurate diagnosis and subsequent treatment of headaches. In addition to the treatment- and prevention-related measures set forth by the RAND authors, ICSI suggests several providerlevel treatment and prevention-related quality measures that are intended to increase the rate of treatment plans for mild, moderate, and severe headaches for migraine sufferers and to

Table 3. Treatment-Related and Prevention-Related Migraine Quality of Care Indicators*

- Patients with acute mild migraine or tension headache should have tried aspirin, acetaminophen, or other nonsteroidal anti-inflammatory agents before being offered any other medication.

- For patients with acute moderate or severe migraine headache, one of the following should have been tried before any other agent is offered: ketorolac, sumatriptan, diydroergotamine, ergotamine, chlorpromazine, or metoclopramide. According to the DISC study by Lipton, the severity of migraine headache would dictate the first medication that someone takes, called the stratified approach.

- Recurrent moderate or severe tension headaches should be treated with a trial of tricyclic antidepressant agents if there are no medical contraindications to their use.

- Patients experiencing more than 2 moderate-to-severe migraine headaches per month, should be offfered prophylactic treatment with one of the following: beta-blockers, calcium channel blockers, topiramate, tricyclic antidepressants, naproxen, aspirin, fluoxetine, valproate, or cyproheptadine. Angiotensin II receptor blockers have also demonstrated efficacy in the prevention of migraines. (Table 4 summarizes the level of evidence of pharmacological agents for migraine prophylaxis according to the US Headache Consortium Guidelines for Migraine Prophylaxis.)

- Sumatriptan and ergotamine should not be administered concurrently.

- Opioid agonists and barbiturates should not be first-line therapy for migraine or tension headaches, except in patients where more specific agents are medically contraindicated.

- Sumatriptan and ergotamine should not be given to patients with a history of uncontrolled hypertension, ischemic heart disease, or angina.

- Percentage of migraine sufferers with treatment plans for mild, moderate, and severe headaches.

- Percentage of migraine sufferers with a treatment plan who adhere to that plan for mild, moderate, and severe headaches.

- Number of prescriptions filled with narcotics or barbiturates for the treatment of migraine.

- Percentage of migraine sufferers with a prescription of narcotics or barbiturates for the treatment of migraine.

- Percentage of migraine sufferers with documented education.

- Percentage of migraine sufferers prescribed appropriate prophylactic treatment based on headache type.

- Percentage of migraine sufferers prescribed appropriate acute treatment based on level of severity.

- Percentage of migraine sufferers prescribed appropriate prophylactic treatment based on level of severity.

*Adapted from references 42, 44-48. 


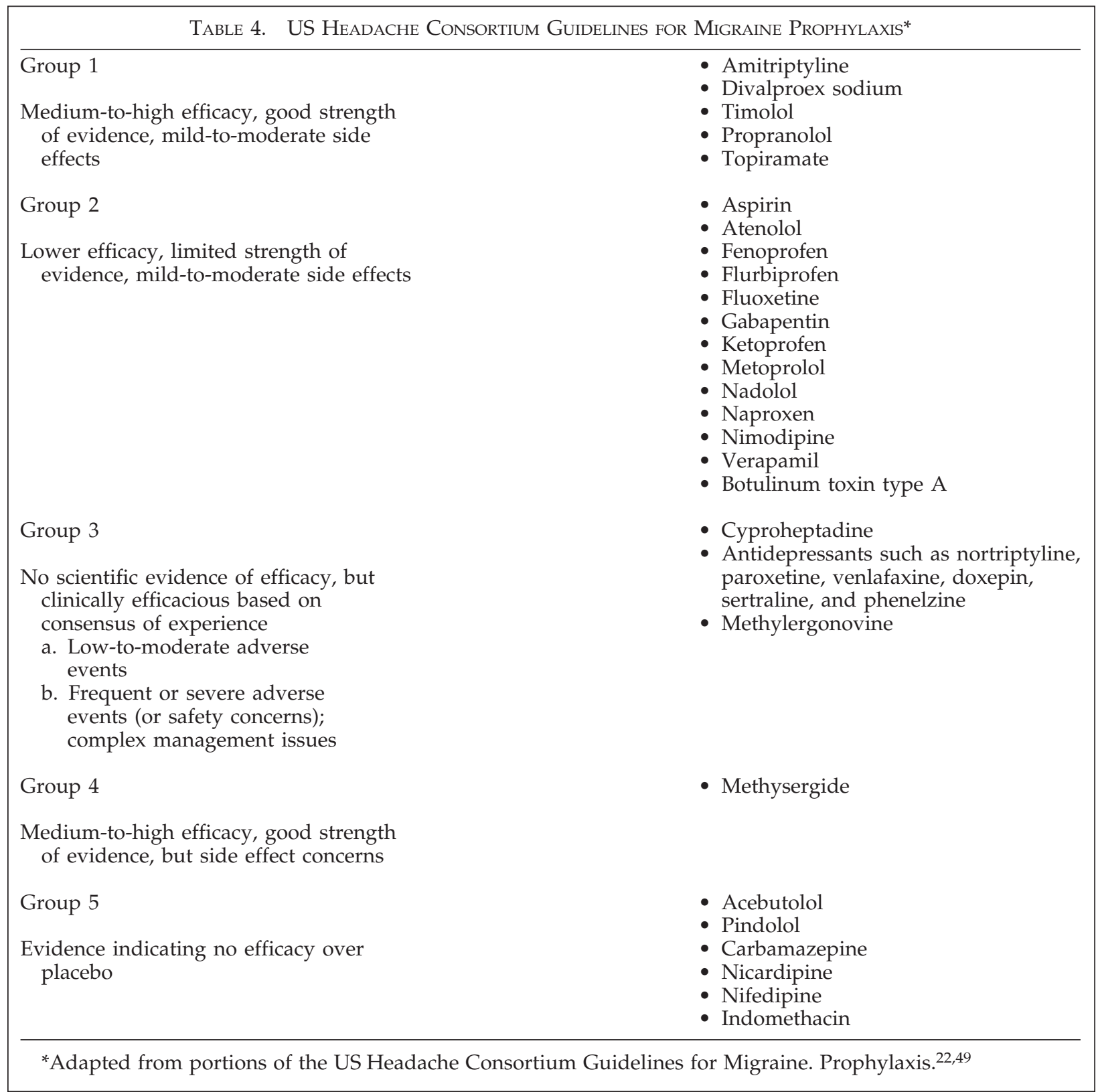

reduce the use of narcotics and barbiturates for treatment (Table 3).

\section{DISCUSSION}

Few migraine care quality measures exist, and those that are available are not easily implemented at the health-plan level. The RAND Corporation collected exisiting quality indicators and ICSI has developed provider-level quality measures. RAND researchers conducted a literature review and sought expert opinion during the development of a comprehensive measure- ment set for headache, which was subsequently used to study, as part of a much larger compilation of quality measures, the quality of care delivered to adults in the United States. Aside from these, few indicators exist. Most RAND study indicators and the suggested ICSI measures were intended for provider-level quality assessment and require chart review. They are not based on administrative data such as claims. Mandating a health-plan quality measure based on chart review creates a significant burden. An alternative approach is to include patient self-reported measures. However, these focus mainly on symptoms and impact on humanistic outcomes (eg, 
functionality and quality of life) and do not usually lend themselves to health-plan level quality assessment. Surveys also may be more difficult for a health plan to implement than measures based on existing health-plan claims data.

Existing migraine disease management models have relied on humanistic outcomes to assess their effectiveness. While patient-reported outcomes are important, administation of surveys may not be feasible for program providers and the outcomes assessment may not be relevant to the health-plan customers. A quality measurement set needs to be based on agreedupon standards of care and expected outcomes. Cost, feasibility, and relevance of the measures to health care purchasers must be considered. A plan-level migraine quality measure set is needed and would benefit disease management providers offering migraine programs. While a good range of measures exist, significant effort is needed to determine what and how to measure quality at the plan level.

\section{ACKNOWLEDGMENT}

This work was supported by Ortho-McNeil Neurologics.

\section{REFERENCES}

1. Linet MS, Stewart WF, Celentano DD, Ziegler D, Sprecher M. An epidemiologic study of headache among adolescents and young adults. IAMA. 1989; 261:2211-2216.

2. Diamond S, Silberstein S, Loder E, Reed ML, Bigal ME, Lipton RB. Patterns of diagnosis and acute and preventive treatment for migraine in the United States: Results from the American Migraine Prevalence and Prevention (AMPP) study. Cephalgia. In press.

3. Stewart WF, Lipton RB, Celentano DD, Reed ML. Prevalence of migraine headache in the United States. Relation to age, income, race, and other sociodemographic factors. JAMA. 1992;267:64-69.

4. Lipton RB, Scher AI, Kolodner K, Liberman J, Steiner TJ, Stewart WF. Migraine in the United States: Epidemiology and patterns of health care use. Neurology. 2002;58:885-894.

5. Hu XH, Markson LE, Lipton RB, Stewart WF, Berger ML. Burden of migraine in the united states: Disability and economic costs. Arch Intern Med. 1999;159: 813-818.

6. Hawkins K, Rupnow M, Wang S. Direct cost burden of migraine among members of US employers. Poster presented at: Annual Meeting of the American Headache Society; June 2006; Los Angeles, CA.

7. Osterhaus JT, Gutterman DL, Plachetka JR. Health care resource and lost labour costs of migraine headache in the US. Pharmacoeconomics. 1992;2:67-76.

8. Hawkins K, Wang S, Rupnow M. Indirect cost burden of migraine among several large US employers. Poster presented at: Annual Meeting of the American Headache Society; June 2006; Los Angeles, CA.

9. Solomon GD, Price KL. Burden of migraine. A review of its socioeconomic impact. Pharmacoeconomics. 1997; 11(suppl 1):1-10.

10. Solomon GD, Litaker DG. The impact of drug therapy on quality of life in headache and migraine. Pharmacoeconomics. 1997;11:334-342.

11. Edmeads J, Findlay H, Tugwell P, Pryse-Phillips W, Nelson RF, Murray TJ. Impact of migraine and tension-type headache on life-style, consulting behaviour, and medication use: A Canadian population survey. Can J Neurol Sci. 1993;20:131-137.

12. Michel P, Dartigues JF, Duru G, Moreau J, Salamon $\mathrm{R}$, Henry P. Incremental absenteeism due to headaches in migraine: Results from the MIG-access French national cohort. Cephalalgia. 1999;19:503-510.

13. Goetzel RZ, Long SR, Ozminkowski RJ, Hawkins K, Wang S, Lynch W. Health, absence, disability, and presenteeism cost estimates of certain physical and mental health conditions affecting U.S. employers. I Occup Environ Med. 2004;46:398-412.

14. National Committee for Quality Assurance. HEDIS 2006. Washington, DC: National Committee for Quality Assurance; 2006.

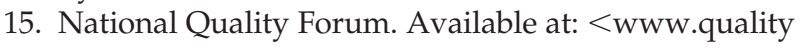
forum.org $>$. Accessed April 10, 2007.

16. Ambulatory Care Quality Alliance. Available at: <www.aqaalliance.org>. Accessed April 10, 2007.

17. Lipton RB, Silberstein SD, Stewart WF. An update on the epidemiology of migraine. Headache. 1994;34:319328.

18. Warshaw LJ, Burton WN. Cutting the costs of migraine: Role of the employee health unit. LOccup Environ Med. 1998;40:943-953.

19. Warshaw LJ, Burton WN, Schneider WJ. Role of the workplace in migraine disease management. Dis Manage Health Outcomes. 2001;9:99-115.

20. Campinha-Bacote DL, Kendle JB, Jones C, et al. Impact of a migraine management program on improving health outcomes. Dis Manage. 2005;8:382-391.

21. Hu HX, Solomon GD, Conboy K, Deml L, Markson LE. Impact of a migraine disease management program: Humanistic outcomes. Dis Manage Health Outcomes. 2004;12:273-280.

22. Silberstein SD, Rosenberg J. Multispecialty consensus on diagnosis and treatment of headache. Neurology. 2000;54:1553.

23. Committee on Quality of Health Care in America, Institute of Medicine. Crossing the Quality Chasm: A New Health System for the 21 ${ }^{\text {st }}$ Century. Washington, DC: National Academies Press; 2001.

24. Donabedian A. The quality of care. How can it be assessed? IAMA. 1988;260:1743-1748. 
25. Perrin EB. Some thoughts on outcomes research, quality improvement, and performance measurement. Med Care. 2002 Jun;40(6 suppl):III89-III91.

26. Hartmaier SL, Santanello NC, Epstein RS, Silberstein SD. Development of a brief 24-hour migraine-specific quality of life questionnaire. Headache. 1995;35:320329.

27. Santanello NC, Hartmaier SL, Epstein RS, Silberstein SD. Validation of a new quality of life questionnaire for acute migraine headache. Headache. 1995;35:330337.

28. Wagner TH, Patrick DL, Galer BS, Berzon RA. A new instrument to assess the long-term quality of life effects from migraine: Development and psychometric testing of the MSQOL. Headache. 1996;36:484-492.

29. Jhingran P, Davis SM, LaVange LM, Miller DW, Helms RW. MSQ: Migraine-specific quality-of-life questionnaire. Further investigation of the factor structure. Pharmacoeconomics. 1998;13:707-717.

30. Jhingran P, Osterhaus JT, Miller DW, Lee JT, Kirchdoerfer L. Development and validation of the migraine-specific quality of life questionnaire. Headache. 1998;38:295-302.

31. Stewart WF, Lipton RB, Kolodner K, Liberman J, Sawyer J. Reliability of the migraine disability assessment score in a population-based sample of headache sufferers. Cephalalgia. 1999;19:107-114;discussion 74.

32. Stewart WF, Lipton RB, Whyte J, et al. An international study to assess reliability of the migraine disability assessment (MIDAS) score. Neurology. 1999;53: 988-994.

33. Jacobson GP, Ramadan NM, Aggarwal SK, Newman CW. The Henry Ford hospital headache disability inventory (HDI). Neurology. 1994;44:837-842.

34. Jacobson GP, Ramadan NM, Norris L, Newman CW. Headache disability inventory (HDI): Short-term testretest reliability and spouse perceptions. Headache. 1995;35:534-539.

35. Stewart WF, Lipton RB, Simon D, Liberman J, Von Korff M. Validity of an illness severity measure for headache in a population sample of migraine sufferers. Pain. 1999;79:291-301.

36. Stewart WF, Lipton RB, Simon D, Von Korff M, Liberman J. Reliability of an illness severity measure for headache in a population sample of migraine sufferers. Cephalalgia. 1998;18:44-51.

37. Bjorner JB, Kosinski M, Ware JE, Jr. Using item response theory to calibrate the headache impact test (HIT) to the metric of traditional headache scales. Qual Life Res. 2003;12:981-1002.

38. Kosinski M, Bayliss MS, Bjorner JB, et al. A six-item short-form survey for measuring headache impact: The HIT-6. Qual Life Res. 2003;12:963-974.

39. Bjorner JB, Kosinski M, Ware JE, Jr. Calibration of an item pool for assessing the burden of headaches: An application of item response theory to the headache impact test (HIT). Qual Life Res. 2003;12:913-933.

40. Chatterton ML, Lofland JH, Shechter A, et al. Reliability and validity of the migraine therapy assessment questionnaire. Headache. 2002;42:1006-1015.
41. Dowson AJ, D'Amico D, Tepper SJ, Baos V, Baudet F, Kilminster S. Identifying patients who require a change in their current acute migraine treatment: The migraine assessment of current therapy (migraineACT) questionnaire. Neurol Sci. 2004;25 (suppl 3): S276-S278.

42. Institute of Clinical Systems Improvement. Health Care Guideline: Diagnosis and treatment of headache. Available at: <www.icsi.org/guidelines_and_more/ guidelines__order_sets___protocols/other_health_ care_conditions/headache/headache__diagnosis_ and_treatment_of_guideline_.html $>$. Accessed April 10, 2007.

43. McGlynn EA, Asch SM, Adams J, et al. The quality of health care delivered to adults in the United States. $\underline{N}$ Engl I Med. 2003;348:2635-2645.

44. Lapuerta P, Asch S. Headache. In: McGlynn EA, Kerr EA, Damberg CL, Asch SM, eds. Quality of Care for Women: A Review of Selected Clinical Conditions and Quality Indicators. Santa Monica, CA: RAND Corporation; 2000.

45. Lapuerta P, Asch S, Clark K. Headache. In: Kerr EA, Asch SM, Hamilton EG, McGlynn EA, eds. Quality of Care for General Medical Conditions: A Review of the Literature and Quality Indicators. Santa Monica, CA: RAND Corporation; 2000.

46. Lipton RB, Stewart WF, Stone AM, Lainez MJ, Sawyer JP. Disability in Strategies of Care Study group. Stratified care vs step care strategies for migraine: The disability in strategies of care (DISC) study: A randomized trial. $\lfloor A M A$. 2000;284:2599-2605.

47. Sculpher M, Millson D, Meddis D, Poole L. Cost-effectiveness analysis of stratified versus stepped care strategies for acute treatment of migraine: The disability in strategies for care (DISC) study. Pharmacoeconomics. 2002;20:91-100.

48 Tronvik E, Stovner LJ, Helde G, Sand T, Bovim G. Prophylactic treatment of migraine with an angiotensin II receptor blocker: A randomized controlled trial. IAMA. 2003;289:65-69.

49. Kaniecki R, Lucas S. Treatment of primary headache: preventive treatment of migraine. In: Standards of Care for Headache Diagnosis and Treatment. Chicago,IL: National Headache Foundation; 2004:40-52.

50. Lipton RB, Dodick D, Sadovsky R, et al. A self-administered screener for migraine in primary care: The ID migraine validation study. Neurology. 2003;61:375382.

51. Cady RK, Borchert LD, Spalding W, Hart CC, Sheftell FD. Simple and efficient recognition of migraine with 3-question headache screen. Headache. 2004;44:323327.

Address reprint requests to: Joshua J. Gagne, Pharm.D.

Ortho-McNeil Janssen Scientific Affairs, LLC 1125 Trenton-Harbourton Road Titusville, NJ 08560

E-mail: jgagne1@omjus.jnj.com 
This article has been cited by:

1. Brian F. Leas, Joshua J. Gagne, Neil I. Goldfarb , Marcia F.T. Rupnow, Stephen Silberstein . 2008. Assessing Quality of Care for Migraineurs: A Model Health Plan Measurement Set. Population Health Management 11:4, 203-208. [Abstract] [PDF] [PDF Plus] 